\title{
Expansive Mappings and Their Applications in Modular Space
}

\author{
A. Azizi, R. Moradi, and A. Razani \\ Department of Mathematics, Faculty of Science, Imam Khomeini International University, Qazvin 34149-16818, Iran \\ Correspondence should be addressed to A. Razani; razani@ipm.ir
}

Received 19 September 2013; Revised 30 January 2014; Accepted 31 January 2014; Published 14 April 2014

Academic Editor: Mohamed Amine Khamsi

Copyright (C) 2014 A. Azizi et al. This is an open access article distributed under the Creative Commons Attribution License, which permits unrestricted use, distribution, and reproduction in any medium, provided the original work is properly cited.

Some fixed point theorems for $\rho$-expansive mappings in modular spaces are presented. As an application, two nonlinear integral equations are considered and the existence of their solutions is proved.

\section{Introduction}

Let $(X, d)$ be a metric space and $B$ a subset of $X$. A mapping $T: B \rightarrow X$ is said to be expansive with a constant $k>1$ such that

$$
d(T x, T y) \geq k d(x, y) \quad \forall x, y \in B .
$$

Xiang and Yuan [1] state a Krasnosel'skii-type fixed point theorem as follows.

Theorem 1 (see [1]). Let $(X,\|\cdot\|)$ be a Banach space and $K \subset X$ a nonempty, closed, and convex subset. Suppose that $T$ and $S$ map $K$ into $X$ such that

(I) $S$ is continuous; $S(K)$ resides in a compact subset of $X$;

(II) $T$ is an expansive mapping;

(III) $z \in S(K)$ implies that $T(K)+z \supset K$, where $T(K)+z=$ $\{y+z \mid y \in T(K)\}$.

Then there exists a point $x^{*} \in K$ with $S x^{*}+T x^{*}=x^{*}$.

For other related results, see also $[2,3]$.

In this paper, we study some fixed point theorems for $S+T$, where $T$ is $\rho$-expansive and $S(B)$ resides in a compact subset of $X_{\rho}$, where $B$ is a closed, convex, and nonempty subset of $X_{\rho}$ and $T, S: B \rightarrow X_{\rho}$. Our results improve the classical version of Krasnosel'skii fixed point theorems in modular spaces.

Finally, as an application, we study the existence of a solution of some nonlinear integral equations in modular function spaces.

In order to do this, first, we recall the definition of modular space (see [4-6]).
Definition 2. Let $X$ be an arbitrary vector space over $K=(\mathbb{R}$ or $\mathbb{C}$ ). Then we have the following.

(a) A functional $\rho: X \rightarrow[0, \infty]$ is called modular if

(i) $\rho(x)=0$ if and only if $x=0$;

(ii) $\rho(\alpha x)=\rho(x)$ for $\alpha \in K$ with $|\alpha|=1$, for all $x \in X$;

(iii) $\rho(\alpha x+\beta y) \leq \rho(x)+\rho(y)$ if $\alpha, \beta \geq 0, \alpha+\beta=1$, for all $x, y \in X$.

If (iii) is replaced by

(iii) ${ }^{\prime} \rho(\alpha x+\beta y) \leq \alpha \rho(x)+\beta \rho(y)$ for $\alpha, \beta \geq 0, \alpha+\beta=$ 1 , for all $x, y \in X$, then the modular $\rho$ is called a convex modular.

(b) A modular $\rho$ defines a corresponding modular space, that is, the space $X_{\rho}$ given by

$$
X_{\rho}=\{x \in X \mid \rho(\alpha x) \longrightarrow 0 \text { as } \alpha \longrightarrow 0\} .
$$

(c) If $\rho$ is convex modular, the modular $X_{\rho}$ can be equipped with a norm called the Luxemburg norm defined by

$$
\|x\|_{\rho}=\inf \left\{\alpha>0 ; \rho\left(\frac{x}{\alpha}\right) \leq 1\right\} .
$$

Remark 3. Note that $\rho$ is an increasing function. Suppose that $0<a<b$; then property (iii), with $y=0$, shows that $\rho(a x)=$ $\rho((a / b)(b x)) \leq \rho(b x)$. 
Definition 4. Let $X_{\rho}$ be a modular space. Then we have the following.

(a) A sequence $\left(x_{n}\right)_{n \in \mathbb{N}}$ in $X_{\rho}$ is said to be

(i) $\rho$-convergent to $x$ if $\rho\left(x_{n}-x\right) \rightarrow 0$ as $n \rightarrow \infty$;

(ii) $\rho$-Cauchy if $\rho\left(x_{n}-x_{m}\right) \rightarrow 0$ as $n, m \rightarrow \infty$.

(b) $X_{\rho}$ is $\rho$-complete if every $\rho$-Cauchy sequence is $\rho$ convergent.

(c) A subset $B \subset X_{\rho}$ is said to be $\rho$-closed if for any sequence $\left(x_{n}\right)_{n \in \mathbb{N}} \subset B$ and $x_{n} \rightarrow x$ then $x \in B$.

(d) A subset $B \subset X_{\rho}$ is called $\rho$-bounded if $\delta_{\rho}(B)=$ $\sup \rho(x-y)<\infty$, for all $x, y \in B$, where $\delta_{\rho}(B)$ is called the $\rho$-diameter of $B$.

(e) $\rho$ has the Fatou property if

$$
\rho(x-y) \leq \liminf \rho\left(x_{n}-y_{n}\right) \text {, }
$$

whenever $x_{n} \rightarrow x$ and $y_{n} \rightarrow y$ as $n \rightarrow \infty$.

(f) $\rho$ is said to satisfy the $\Delta_{2}$-condition if $\rho\left(2 x_{n}\right) \rightarrow 0$ whenever $\rho\left(x_{n}\right) \rightarrow 0$ as $n \rightarrow \infty$.

\section{Expansive Mapping in Modular Space}

In 2005, Hajji and Hanebaly [7] presented a modular version of Krasnosel'skii fixed point theorem, for a $\rho$-contraction and a $\rho$-completely continuous mapping.

Using the same argument as in [1], we state the modular version of Krasnosel'skii fixed point theorem for $S+T$, where $T$ is a $\rho$-expansive mapping and the image of $B$ under $S$; that is, $S(B)$ resides in a compact subset of $X_{\rho}$, where $B$ is a subset of $X_{\rho}$.

Due to this, we recall the following definitions and theorems.

Definition 5. Let $X_{\rho}$ be a modular space and $B$ a nonempty subset of $X_{\rho}$. The mapping $T: B \rightarrow X_{\rho}$ is called $\rho$-expansive mapping, if there exist constants $c, k, l \in \mathbb{R}^{+}$such that $c>l$, $k>1$ and

$$
\rho(l(T x-T y)) \geq k \rho(c(x-y)),
$$

for all $x, y \in B$.

Example 6. Let $X_{\rho}=B=\mathbb{R}^{+}$and consider $T: B \rightarrow B$ with $T x=x^{n}+4 x+5$ for $x \in B$ and $n \in \mathbb{N}$. Then for all $x, y \in B$, we have

$$
\begin{aligned}
|T x-T y| & =\left|x^{n}-y^{n}+4(x-y)\right| \\
& =\left|(x-y)\left(x^{n-1}+y x^{n-2}+\cdots+y^{n-1}\right)+4(x-y)\right| \\
& \geq 4|x-y| .
\end{aligned}
$$

Therefore $T$ is an expansive mapping with constant $k=4$.
Theorem 7 (Schauder's fixed point theorem, page 825; see [1, $8])$. Let $(X,\|\cdot\|)$ be a Banach space and $K \subset X$ is a nonempty, closed, and convex subset. Suppose that the mapping $S: K \rightarrow$ $K$ is continuous and $S(K)$ resides in a compact subset of $X$. Then $S$ has at least one fixed point in $K$.

We need the following theorem from $[6,9]$.

Theorem 8 (see $[6,9]$ ). Let $X_{\rho}$ be a $\rho$-complete modular space. Assume that $\rho$ is a convex modular satisfying the $\Delta_{2}$-condition and $B$ is a nonempty, $\rho$-closed, and convex subset of $X_{\rho} . T$ : $B \rightarrow B$ is a mapping such that there exist $c, k, l \in \mathbb{R}^{+}$such that $c>l, 0<k<1$ and for all $x, y \in B$ one has

$$
\rho(c(T x-T y)) \leq k \rho(l(x-y)) .
$$

Then there exists a unique fixed point $z \in B$ such that $T z=z$.

Theorem 9. Let $X_{\rho}$ be a $\rho$-complete modular space. Assume that $\rho$ is a convex modular satisfying the $\Delta_{2}$-condition and $B$ is a nonempty, $\rho$-closed, and convex subset of $X_{\rho} . T: B \rightarrow X_{\rho}$ is a $\rho$-expansive mapping satisfying inequality (5) and $B \subset T(B)$. Then there exists a unique fixed point $z \in B$ such that $T z=z$.

Proof. We show that operator $T$ is a bijection from $B$ to $T(B)$. Let $x_{1}$ and $x_{2}$ be in $B$ such that $T x_{1}=T x_{2}$; by inequality (5), we have $x_{1}=x_{2}$; also since $B \subset T(B)$ it follows that the inverse of $T: B \rightarrow T(B)$ exists. For all $x, y \in T(B)$,

$$
\rho(c(f x-f y)) \leq \frac{1}{k} \rho(l(x-y)),
$$

where $f=T^{-1}$. We consider $f=\left.T^{-1}\right|_{B}: B \rightarrow B$, where $\left.T^{-1}\right|_{B}$ denotes the restriction of the mapping $T^{-1}$ to the set $B$. Since $B \subset T(B)$, then $f$ is a $\rho$-contraction. Also since $B$ is a $\rho$ closed subset of $X_{\rho}$, then, by Theorem 8 , there exists a $z \in B$ such that $f z=z$. Also $z$ is a fixed point of $T$.

For uniqueness, let $z$ and $w$ be two arbitrary fixed points of $T$; then

$$
\begin{aligned}
\rho(c(z-w)) & \geq \rho(l(z-w))=\rho(l(T z-T w)) \\
& \geq k \rho(c(z-w)) ;
\end{aligned}
$$

hence $(k-1) \rho(c(z-w)) \leq 0$ and $z=w$.

We need the following lemma for the main result.

Lemma 10. Suppose that all conditions of Theorem 9 are fulfilled. Then the inverse of $f:=I-T: B \rightarrow(I-T)(B)$ exists and

$$
\rho\left(c\left(f^{-1} x-f^{-1} y\right)\right) \leq \frac{1}{k-1} \rho\left(l^{\prime}(x-y)\right),
$$

for all $x, y \in f(B)$, where $l^{\prime}=\alpha$ and $\alpha$ is conjugate of $c / l$; that is, $(l / c)+(1 / \alpha)=1$ and $c>2 l$.

Proof. For all $x, y \in B$,

$$
\begin{aligned}
& \rho(l(T x-T y))=\rho(l((x-f x)-(y-f y))) \\
& \leq \rho(c(x-y))+\rho(\alpha l(f x-f y)) ; \\
& k \rho(c(x-y))-\rho(c(x-y)) \leq \rho(\alpha l(f x-f y)),
\end{aligned}
$$


then

$$
(k-1) \rho(c(x-y)) \leq \rho\left(l^{\prime}(f x-f y)\right) .
$$

Now, we show that $f$ is an injective operator. Let $x, y \in B$ and $f x=f y$; then by inequality $(12),(k-1) \rho(c(x-y)) \leq 0$ and $x=y$. Therefore $f$ is an injective operator from $B$ into $f(B)$, and the inverse of $f: B \rightarrow f(B)$ exists. Also for all $x, y \in f(B)$, we have $f^{-1} x, f^{-1} y \in B$. Then for all $x, y \in f(B)$, by inequality (12) we get

$$
\rho\left(c\left(f^{-1} x-f^{-1} y\right)\right) \leq \frac{1}{k-1} \rho\left(l^{\prime}(x-y)\right) .
$$

Theorem 11. Let $X_{\rho}$ be a $\rho$-complete modular space. Assume that $\rho$ is a convex modular satisfying the $\Delta_{2}$-condition and $B$ is a nonempty, $\rho$-closed, and convex subset of $X_{\rho}$. Suppose that

(I) $S: B \rightarrow X_{\rho}$ is a $\rho$-continuous mapping and $S(B)$ resides in a $\rho$-compact subset of $X_{\rho}$;

(II) $T: B \rightarrow X_{\rho}$ is a $\rho$-expansive mapping satisfying inequality (5) such that $c>2 l$;

(III) $x \in S(B)$ implies that $B C x+T(B)$, where $T(B)+x=$ $\{y+x \mid y \in T(B)\}$.

There exists a point $z \in B$ such that $S z+T z=z$.

Proof. Let $w \in S(B)$ and $T_{w}=T+w$. Consider the mapping $T_{w}: B \rightarrow X_{\rho}$; then by Theorem 9 , the equation $T x+w=x$ has a unique solution $x=\eta(w)$. Now, we show that $\eta$ is a $\rho$-contraction. For $w_{1}, w_{2} \in S(B), T\left(\eta\left(w_{1}\right)\right)+w_{1}=\eta\left(w_{1}\right)$ and $T\left(\eta\left(w_{2}\right)\right)+w_{2}=\eta\left(w_{2}\right)$. Applying the same technique in Lemma 10 ,

$$
(k-1) \rho\left(c\left(\eta\left(w_{1}\right)-\eta\left(w_{2}\right)\right)\right) \leq \rho\left(l^{\prime}\left(w_{1}-w_{2}\right)\right),
$$

where $l^{\prime}=\alpha l$. Then

$$
\rho\left(c\left(\eta\left(w_{1}\right)-\eta\left(w_{2}\right)\right)\right) \leq \frac{1}{k-1} \rho\left(l^{\prime}\left(w_{1}-w_{2}\right)\right) .
$$

Therefore, mapping $\eta: S(B) \rightarrow B$ is a $\rho$-contraction and hence is a $\rho$-continuous mapping. By condition (I), $\eta S: B \rightarrow$ $B$ is also $\rho$-continuous mapping and, by $\Delta_{2}$-condition, $\eta S$ is $\|\cdot\|_{\rho}$-continuous mapping. Also $\eta S(B)$ resides in a $\|\cdot\|_{\rho}$ compact subset of $X_{\rho}$. Then using Theorem 7, there exists a $z \in B$ such that $z=\eta(S(z))$ which implies that $T z+S z=$ $z$.

The following theorem is another version of Theorem 11.

Theorem 12. Let $X_{\rho}$ be a $\rho$-complete modular space. Assume that $\rho$ is a convex modular satisfying the $\Delta_{2}$-condition and $B$ is a nonempty, $\rho$-closed, and convex subset of $X_{\rho}$. Suppose that

(I) $S: B \rightarrow X_{\rho}$ is a $\rho$-continuous mapping and $S(B)$ resides in a $\rho$-compact subset of $X_{\rho}$;

(II) $T: B \rightarrow X_{\rho}$ or $T: X_{\rho} \rightarrow X_{\rho}$ is a $\rho$-expansive mapping satisfying inequality (5) such that $c>2 l$;

(III) $S(B) \subset(I-T)\left(X_{\rho}\right)$ and $[x=T x+S y, y \in B$ implies that $x \in B]$ or $S(B) \subset(I-T)(B)$.

Then there exists a point $z \in B$ such that $S z+T z=z$.
Proof. By condition (III), for each $w \in B$, there exists $x \in X_{\rho}$ such that $x-T x=S w$. If $S(B) \subset(I-T)(B)$, then $x \in B$; if $S(B) \subset(I-T)\left(X_{\rho}\right)$, then by Lemma 10 and condition (III), $x=(I-T)^{-1} S w \in B$. Now $(I-T)^{-1}$ is a $\rho$-continuous and so $(I-T)^{-1} S$ is a $\rho$-continuous mapping of $B$ into $B$. Since $S(B)$ resides in a $\rho$-compact subset of $X_{\rho}$, so $(I-T)^{-1} S(B)$ resides in a $\rho$-compact subset of the closed set $B$. By using Theorem 7 , there exists a fixed point $z \in B$ such that $z=(I-T)^{-1} S z$.

Using the same argument as in [2], we can state a new version of Theorem 11, where $S$ is $\rho$-sequentially continuous.

Definition 13. Let $X_{\rho}$ be a modular space and $B$ a subset of $X_{\rho}$. A mapping $T: B \rightarrow X_{\rho}$ is said to be

(1) $\rho$-sequentially continuous on the set $B$ if for every sequence $\left\{x_{n}\right\} \subset B$ and $x \in B$ such that $\rho\left(x_{n}-x\right) \rightarrow 0$, then $\rho\left(T x_{n}-T x\right) \rightarrow 0$;

(2) $\rho$-closed if for every sequence $\left\{x_{n}\right\} \subset B$ such that $\rho\left(x_{n}-x\right) \rightarrow 0$ and $\rho\left(T x_{n}-y\right) \rightarrow 0$, then $T x=y$.

Definition 14. Let $X_{\rho}$ be a modular space and $B, C$ two subsets of $X_{\rho}$. Suppose that $T: B \rightarrow X_{\rho}$ and $S: C \rightarrow X_{\rho}$ are two mappings. Define

$$
F=\{x \in B: x=T x+S y \text { for some } y \in C\} .
$$

Theorem 15. Let $X_{\rho}$ be a $\rho$-complete modular space. Assume that $\rho$ is a convex modular satisfying the $\Delta_{2}$-condition and $B$ is a nonempty, $\rho$-closed, and convex subset of $X_{\rho}$. Suppose that

(I) $S: B \rightarrow X_{\rho}$ is $\rho$-sequentially continuous;

(II) $T: B \rightarrow X_{\rho}$ is a $\rho$-expansive mapping satisfying inequality (5) such that $c>2 l$;

(III) $x \in S(B)$ implies that $B C x+T(B)$, where $T(B)+x=$ $\{y+x \mid y \in T(B)\}$;

(IV) $T$ is $\rho$-closed in $F$ and $F$ is relatively $\rho$-compact.

Then there exists a point $z \in B$ such that $S z+T z=z$.

Proof. Let $w \in B$, and $T_{S w}=T+S w$. One considers the mapping $T_{S w}: B \rightarrow X_{\rho}$; by Theorem 9 , the equation

$$
T x+S w=x
$$

has a unique solution $x=\eta(S w) \in B$.

Now, we show that $\eta S=(I-T)^{-1}$ exists. For any $w_{1}, w_{2} \in$ $B$ and by the same technique of Lemma 10, we have

$$
\rho\left(c\left(\eta\left(S w_{1}\right)-\eta\left(S w_{2}\right)\right)\right) \leq \frac{1}{k-1} \rho\left(l^{\prime}\left(w_{1}-w_{2}\right)\right),
$$

where $l^{\prime}=\alpha l$. This implies that $\eta S=(I-T)^{-1}$ exists and for all $w \in B, \eta S w=(I-T)^{-1} S w$ and $\eta S(B) \subset F$.

We show that $\eta S$ is $\rho$-sequentially continuous in $B$. Let $\left\{x_{n}\right\}$ be a sequence in $B$ and $x \in B$ such that $\rho\left(x_{n}-x\right) \rightarrow 0$. Since $\eta S\left(x_{n}\right) \in F$ and $F$ is relatively $\rho$-compact, then there exists $z \in B$ such that $\rho\left(\eta S x_{n}-z\right) \rightarrow 0$. On the other hand, by condition (I), $\rho\left(S x_{n}-S x\right) \rightarrow 0$. Thus by (17), we get

$$
T\left(\eta S x_{n}\right)+S x_{n}=\eta S x_{n} ;
$$


then

$$
\begin{aligned}
\rho\left(\frac{T\left(\eta S x_{n}\right)-(z-S x)}{2}\right) & =\rho\left(\frac{\left(\eta S x_{n}-S x_{n}\right)-(z-S x)}{2}\right) \\
& \leq \rho\left(\eta S x_{n}-z\right)+\rho\left(S x_{n}-S x\right)
\end{aligned}
$$

therefore when $n \rightarrow \infty$, condition (IV) implies that $T z=$ $z-S x$; that is, $z=\eta S x$ and

$$
\rho\left(\eta S x_{n}-\eta S x\right) \longrightarrow 0
$$

then $\eta S$ is $\rho$-sequentially continuous in $F$. By $\Delta_{2}$-condition, $\eta S$ is $\|\cdot\|_{\rho}$-sequentially continuous. Let $H=\overline{\mathrm{co}}^{\|} \cdot \|_{\rho} F$, where $\overline{\mathrm{co}}^{\|\cdot\|_{\rho}}$ denotes the closure of the convex hull in the sense of $\|\cdot\|_{\rho}$. Then $H \subset B$ and is a compact set. Therefore $\eta S$ is $\|\cdot\|_{\rho}$-sequentially continuous from $H$ into $H$. Then using Theorem 7, $\eta S$ has a fixed point $z \in H$ such that $\eta S z=z$. From (17), we have

$$
T(\eta S z)+S z=\eta S z
$$

that is, $T z+S z=z$.

The following theorem is another version of Theorem 15.

Theorem 16. Let $X_{\rho}$ be a $\rho$-complete modular space. Assume that $\rho$ is a convex modular satisfying the $\Delta_{2}$-condition and $B$ is a nonempty, $\rho$-closed, and convex subset of $X_{\rho}$. Suppose that

(I) $S: B \rightarrow X_{\rho}$ is $\rho$-sequentially continuous;

(II) $T: B \rightarrow X_{\rho}$ is a $\rho$-expansive mapping satisfying inequality (5), such that $c>2 l$;

(III) $S(B) \subset(I-T)\left(X_{\rho}\right)$ and $[x=T x+S y, y \in B]$ implies that $x \in B($ or $S(B) \subset(I-T)(B))$.

(IV) $T$ is $\rho$-closed in $F$ and $F$ is relatively $\rho$-compact.

Then there exists a point $z \in B$ such that $S z+T z=z$.

Proof. By (III) for each $w \in B$, there exists $x \in X_{\rho}$ such that $x-T x=S w$ and $x=(I-T)^{-1} S w \in B$. By the same technique of Theorem $15,(I-T)^{-1} S: B \rightarrow B$ is $\rho$ sequentially continuous and there exists a $z \in B$ such that $z=(I-T)^{-1} S z$

\section{Integral Equation for $\rho$-Expansive Mapping in Modular Function Spaces}

In this section, we study the following integral equation:

$$
x(t)=\phi(t, x(t))+\int_{0}^{t} \psi(t, s, x(s)) d s, \quad x \in C\left(I, L^{\varphi}\right),
$$

where $L^{\varphi}$ is the Musielak-Orlicz space and $I=[0, b] \subset \mathbb{R}$. $C\left(I, L^{\varphi}\right)$ denote the space of all $\rho$-continuous functions from $I$ to $L^{\varphi}$ with the modular $\sigma(x)=\sup _{t \in I} \rho(x(t))$. Also $C\left(I, L^{\varphi}\right)$ is a real vector space. If $\rho$ is a convex modular, then $\sigma$ is a convex modular. Also, if $\rho$ satisfies the Fatou property and $\Delta_{2}$-condition, then $\sigma$ satisfies the Fatou property and $\Delta_{2}$ condition (see [9]).

To study the integral equation (23), we consider the following hypotheses.

(1) $\phi: I \times L^{\varphi} \rightarrow L^{\varphi}$ is a $\rho$-expansive mapping; that is, there exist constants $c, k, l \in \mathbb{R}^{+}$such that $c>2 l, k \geq 2$ and for all $x, y \in L^{\varphi}$

$$
\rho(l(\phi(t, x)-\phi(t, y))) \geq k \rho(c(x-y))
$$

and $\phi$ is onto. Also for $t \in I, \phi(t, \cdot): L^{\varphi} \rightarrow L^{\varphi}$ is $\rho$ continuous.

(2) $\psi$ is a function from $I \times I \times L^{\varphi}$ into $L^{\varphi}$ such that $\psi(t, s, \cdot): x \rightarrow \psi(t, s, x)$ is $\rho$-continuous on $L^{\varphi}$ for almost all $t, s \in I$ and $\psi(t, \cdot, x): s \rightarrow \psi(t, s, x)$ is measurable function on $I$ for each $x \in L^{\varphi}$ and for almost all $t \in I$. Also, there are nondecreasing continuous functions $\beta, \gamma: I \rightarrow \mathbb{R}^{+}$such that

$$
\begin{gathered}
\lim _{t \rightarrow \infty} \beta(t) \int_{0}^{t} \gamma(s) d s=0, \\
\rho(c(\psi(t, s, x))) \leq \beta(t) \gamma(s),
\end{gathered}
$$

for all $t, s \in I, s \leq t$ and $x \in L^{\varphi}$.

(3) There exists measurable function $\eta: I \times I \times I \rightarrow \mathbb{R}^{+}$ such that

$$
\rho(\psi(t, s, x)-\psi(r, s, x)) \leq \eta(t, r, s),
$$

for all $t, r, s \in I$ and $x \in L^{\varphi}$; also $\lim _{t \rightarrow r} \int_{0}^{b} \eta(t, r, s) d s=0$.

(4) $\rho(\psi(t, s, x)-\psi(t, s, y)) \leq \rho(x-y)$ for all $t, s \in I$ and $x, y \in L^{\varphi}$.

Remark 17 (see [7]). We consider $L^{\varphi}$, the Musielak-Orlicz space. Since $\rho$ is convex and satisfies the $\Delta_{2}$-condition, then

$$
\left\|x_{n}-x\right\|_{\rho} \longrightarrow 0 \Longleftrightarrow \rho\left(x_{n}-x\right) \longrightarrow 0,
$$

as $n \rightarrow \infty$ on $L^{\varphi}$. This implies that the topologies generated by $\|\cdot\|_{\rho}$ and $\rho$ are equivalent.

Theorem 18. Suppose that the conditions (1)-(4) are satisfied. Further assume that $L^{\varphi}$ satisfies the $\Delta_{2}$-condition. Also $\omega(t)=$ $\beta(t) \int_{0}^{t} \gamma(s) d s$ and $\omega(0)=0 ;$ also $\sup \{\rho(c(\phi(t, v))), t \in I, v \in$ $\left.L^{\varphi}\right\} \leq \omega(t)$. Then integral equation (23) has at least one solution $x \in C\left(I, L^{\varphi}\right)$.

Proof. Suppose that

$$
\begin{gathered}
T x(t)=\phi(t, x(t)), \\
S x(t)=\int_{0}^{t} \psi(t, s, x(s)) d s .
\end{gathered}
$$

Conditions (1) and (2) imply that $T$ and $S$ are well defined on $C\left(I, L^{\varphi}\right)$. Define the set $B=\left\{x \in C\left(I, L^{\varphi}\right) ; \rho(c(x(t))) \leq\right.$ 
$\omega(t)$ for all $t \in I\}$. Then $B$ is a nonempty, $\rho$-bounded, $\rho$-closed, and convex subset of $C\left(I, L^{\varphi}\right)$. Equation (23) is equivalent to the fixed point problem $x=T x+S x$. By Theorem 12, we find the fixed point for $T+S$ in $B$. Due to this, we prove that $S$ satisfies the condition $(I)$ of Theorem 12 . For $x \in B$, we show that $S x \in B$. Indeed,

$$
\begin{aligned}
\rho(c(S x(t))) & =\rho\left(c\left(\int_{0}^{t} \psi(t, s, x(s)) d s\right)\right) \\
& \leq \int_{0}^{t} \rho(c(\psi(t, s, x(s)))) d s \\
& \leq \int_{0}^{t} \beta(t) \gamma(s) d s \\
& =\omega(t) ;
\end{aligned}
$$

then $S x \in B$. Since $S(B) \subset B$ and $B$ is $\rho$-bounded, $S(B)$ is $\sigma$-bounded and by $\Delta_{2}$-condition $\|\cdot\|_{\sigma}$-bounded.

We show that $S(B)$ is $\rho$-equicontinuous. For all $t, r \in I$ and $x \in L^{\varphi}$ such that $t<r$,

$$
S x(t)-S x(r)=\int_{0}^{t} \psi(t, s, x(s)) d s-\int_{0}^{r} \psi(r, s, x(s)) d s ;
$$

then by condition (3),

$$
\rho(S x(t)-S x(r)) \leq \int_{0}^{b} \eta(t, r, s) d s
$$

since $\lim _{t \rightarrow r} \int_{0}^{b} \eta(t, r, s) d s=0$, then $S(B)$ is $\rho$ equicontinuous. By using the Arzela-Ascoli theorem, we obtain that $S$ is a $\sigma$-compact mapping. Next, we show that $S$ is $\sigma$-continuous. Suppose that $\varepsilon>0$ is given; we find a $\delta>0$ such that $\sigma(x-y)<\delta$, for some $x, y \in B$. Note that

$$
S x(t)-S y(t)=\int_{0}^{t} \psi(t, s, x(s)) d s-\int_{0}^{t} \psi(t, s, y(s)) d s ;
$$

also

$$
\rho(S x(t)-S y(t)) \leq \int_{0}^{t} \rho(x(s)-y(s)) d s \leq \int_{0}^{t} \sigma(x-y) d s
$$

then

$$
\sigma(S x-S y) \leq \int_{0}^{b} \sigma(x-y) d s \leq \varepsilon
$$

therefore $S$ is $\sigma$-continuous.

Since $\phi$ is $\rho$-continuous, it shows that $T$ transforms $C\left(I, L^{\varphi}\right)$ into itself. In view of supremum $\rho$ and condition (1), it is easy to see that $T$ is $\sigma$-expansive with constant $k \geq 2$. For $x, y \in B$,

$$
\begin{aligned}
& \rho(l(T x(t)-T y(t))) \\
& \leq \rho(c(x(t)-y(t))) \\
& \quad+\rho(\alpha l((I-T) x(t)-(I-T) y(t))) ;
\end{aligned}
$$

then

$$
\begin{gathered}
\rho(\alpha l((I-T) x(t)-(I-T) y(t))) \\
\geq(k-1) \rho(c(x(t)-y(t))),
\end{gathered}
$$

where $\alpha$ is conjugate of $c / l$. Let $r=\alpha l$; since $k \geq 2$, then

$$
\rho(r(I-T) x(t)) \geq(k-1) \rho(c(x(t))) \geq \rho(c(x(t))) .
$$

Now, assume that $x=T x+S y$ for some $y \in B$. Since $c>2 l$, then $r<c$, and

$$
\begin{aligned}
\rho(c(x(t))) & \leq \rho(r(I-T) x(t))=\rho(r(S y(t))) \\
& \leq \rho(c(S y(t))) \leq \omega(t),
\end{aligned}
$$

which shows that $x \in B$. Now, define a map $T_{z}$ as follows:

$$
T_{z}: C\left(I, L^{\varphi}\right) \longrightarrow C\left(I, L^{\varphi}\right)
$$

for each $z \in C\left(I, L^{\varphi}\right)$; by

$$
T_{z} x(t)=T x(t)+z(t),
$$

for all $x, y \in C\left(I, L^{\varphi}\right)$,

$$
\begin{aligned}
\rho\left(l\left(T_{z} x(t)-T_{z} y(t)\right)\right) & =\rho(l(T x(t)-T y(t))) \\
& \geq k \rho(c(x(t)-y(t))) ;
\end{aligned}
$$

therefore

$$
\sigma\left(l\left(T_{z} x-T_{z} y\right)\right) \geq k \sigma(c(x-y))
$$

then $T_{z}$ is $\sigma$-expansive with constant $k \geq 2$ and $T_{z}$ is onto. By Theorem 9, there exists $w \in C\left(I, L^{\varphi}\right)$ such that $T_{z} w=w$; that is, $(I-T) w=z$. Hence $S(B) \subset(I-T)\left(L^{\varphi}\right)$ and condition (III) of Theorem 12 holds. Therefore by Theorem $12, S+T$ has a fixed point $z \in B$ with $T z+S z=z$; that is, $z$ is a solution to (23).

Now, we consider another integral equation.

Let $L^{\varphi}$ be the Musielak-Orlicz space and $I=[0, b] \subset \mathbb{R}$. Suppose that $\rho$ is convex and satisfies the $\Delta_{2}$-condition. Since topologies generated by $\|\cdot\|_{\rho}$ and $\rho$ are equivalent, then we consider Banach space $\left(L^{\varphi},\|\cdot\|_{\rho}\right)$ and $C\left(I, L^{\varphi}\right)$ denote the space of all $\|\cdot\|_{\rho}$-continuous functions from $I$ to $L^{\varphi}$ with the modular $\|x\|_{\sigma}=\sup _{t \in I}\|x(t)\|_{\rho}$; also $C\left(I, L^{\varphi}\right)$ is a real vector space. Consider the nonlinear integral equation

$$
\begin{array}{r}
x(t)=\phi(t, x(t)) \\
+\lambda(t, x(t)) \int_{0}^{t} \omega(t, s) \psi(s, x(s)) d s, \\
x \in C\left(I, L^{\varphi}\right),
\end{array}
$$

where

(1) $\phi: I \times L^{\varphi} \rightarrow L^{\varphi}$ is a $\|\cdot\|_{\rho}$-expansive mapping; that is, there exists constant $l \geq 2$ such that

$$
\|\phi(t, x)-\phi(t, y)\|_{\rho} \geq l\|x-y\|_{\rho}
$$


for all $x, y \in L^{\varphi}$ and $\phi$ is onto; also for $t \in I, \phi(t, \cdot)$ : $L^{\varphi} \rightarrow L^{\varphi}$ is $\|\cdot\|_{\rho}$-continuous;

(2) $\psi$ is function from $I \times L^{\varphi}$ into $L^{\varphi}$ such that $\psi(t, \cdot)$ : $L^{\varphi} \rightarrow L^{\varphi}$ is a $\|\cdot\|_{\rho}$-continuous and $t \rightarrow \psi(t, x)$ is measurable for every $x \in L^{\varphi}$. Also, there exist functions $\beta \in L^{1}(I)$ and a nondecreasing continuous function $\gamma:[0, \infty) \rightarrow(0, \infty)$ such that

$$
\|\psi(t, x)\|_{\rho} \leq \beta(t) \gamma\left(\|x\|_{\rho}\right)
$$

for all $t \in I$ and $x \in L^{\varphi}$. Also for $t \in I, x \rightarrow \psi(t, x)$ is nondecreasing on $L^{\varphi}$;

(3) $\lambda$ is function from $I \times L^{\varphi}$ into $L^{\varphi}$ such that $\lambda(t, \cdot)$ : $L^{\varphi} \rightarrow L^{\varphi}$ is $\|\cdot\|_{\rho}$-continuous and there exists a $a \geq 0$ such that

$$
\|\lambda(t, x)-\lambda(t, y)\|_{\rho} \leq a\|x-y\|_{\rho}
$$

for all $t \in I$ and $x \in L^{\varphi}$; also for $x \in L^{\varphi}, t \rightarrow \lambda(t, x)$ is nondecreasing on $I$ and for $t \in I, x \rightarrow \lambda(t, x)$ is nondecreasing on $L^{\varphi}$;

(4) $\omega$ is function from $I \times I$ into $\mathbb{R}^{+}$. For each $t \in I, \omega(t, s)$ is measurable on $[0, t]$. Also $\overline{\omega(t)}=\operatorname{esssup}|\omega(t, s)|$ is bounded on $[0, b]$ and $r=\sup |\overline{\omega(t)}|$. The map $\omega(\cdot, s)$ : $t \rightarrow \omega(t, s)$ is continuous from $I$ to $L^{\infty}(I)$. Also for $s \in I, t \rightarrow \omega(t, s)$ is nondecreasing on $I$.

Theorem 19. Suppose that the conditions (1)-(4) are satisfied and there exists a constant $k \geq 0$ such that for all $t \in I$,

$$
\int_{0}^{t} \beta(s) d s<\frac{k}{(a k+h) r b} \int_{0}^{t} \frac{1}{\gamma(k)} d s,
$$

where $h:=\sup \left\{\|\lambda(t, x)\|_{\rho}, t \in I, x \in L^{\varphi}\right\}$ and also $\sup \left\{\|\phi(t, x)\|_{\rho}, t \in I, x \in L^{\varphi}\right\} \leq k$. Then integral equation (43) has at least one solution $x \in C\left(I, L^{\varphi}\right)$.

Proof. Define

$$
B=\left\{x \in C\left(I, L^{\varphi}\right) ;\|x(t)\|_{\rho} \leq k \forall t \in I\right\}
$$

then $B$ is a nonempty, $\|\cdot\|_{\rho}$-bounded, $\|\cdot\|_{\rho}$-closed, and convex subset of $C\left(I, L^{\varphi}\right)$. Consider

$$
\begin{gathered}
T x(t)=\phi(t, x(t)), \\
S x(t)=\lambda(t, x(t)) \int_{0}^{t} \omega(t, s) \psi(s, x(s)) d s .
\end{gathered}
$$

It is easy that by the hypothesis $T$ and $S$ are well defined on $C\left(I, L^{\varphi}\right)$.
For $x \in B$, we show that $S x \in B$. Consider

$\|S x(t)\|_{\rho}$

$=\left\|\lambda(t, x(t)) \int_{0}^{t} \omega(t, s) \psi(s, x(s)) d s\right\|_{\rho}$

$=\left\|(\lambda(t, x(t))-\lambda(t, 0)+\lambda(t, 0)) \int_{0}^{t} \omega(t, s) \psi(s, x(s)) d s\right\|_{\rho}$

$\leq\left(a\|x(t)\|_{\rho}+h\right) r \int_{0}^{t} \beta(s) \gamma\left(\|x(s)\|_{\rho}\right) d s$

$\leq(a k+h) r \int_{0}^{t} \beta(s) \gamma(k) d s$

$\leq(a k+h) r \int_{0}^{b} \frac{k \gamma(k)}{(a k+h) r b \gamma(k)} d s$

$\leq k$.

Let $x \in B$ and assume that $t>\tau \in I$ such that $|t-\tau|<\delta$, for a given positive constant $\delta$. We have

$$
\begin{aligned}
& \|S x(t)-S x(\tau)\|_{\rho} \\
& =\| \lambda(t, x(t)) \int_{0}^{t} \omega(t, s) \psi(s, x(s)) d s \\
& -\lambda(\tau, x(\tau)) \int_{0}^{\tau} \omega(\tau, s) \psi(s, x(s)) d s \|_{\rho} \\
& =\| \lambda(t, x(t)) \int_{0}^{t} \omega(t, s) \psi(s, x(s)) d s \\
& \pm \lambda(t, x(t)) \int_{0}^{t} \omega(\tau, s) \psi(s, x(s)) d s \\
& \pm \lambda(\tau, x(\tau)) \int_{0}^{t} \omega(\tau, s) \psi(s, x(s)) d s \\
& -\lambda(\tau, x(\tau)) \int_{0}^{\tau} \omega(\tau, s) \psi(s, x(s)) d s \|_{\rho} \\
& \leq \| \lambda(t, x(t))\left(\int_{0}^{t} \omega(t, s) \psi(s, x(s)) d s\right. \\
& \left.-\int_{0}^{t} \omega(\tau, s) \psi(s, x(s)) d s\right) \|_{\rho} \\
& +\|(\lambda(\tau, x(\tau))-\lambda(\tau, x(\tau))) \\
& \times \int_{0}^{t} \omega(\tau, s) \psi(s, x(s)) d s \|_{\rho} \\
& +\left\|\lambda(\tau, x(\tau)) \int_{\tau}^{t} \omega(\tau, s) \psi(s, x(s)) d s\right\|_{\rho} ;
\end{aligned}
$$


since

$$
\begin{aligned}
& \| \lambda(t, x(t))\left(\int_{0}^{t} \omega(t, s) \psi(s, x(s)) d s\right. \\
& \left.-\int_{0}^{t} \omega(\tau, s) \psi(s, x(s)) d s\right) \|_{\rho} \\
& =\left\|\lambda(t, x(t))\left(\int_{0}^{t}(\omega(t, s)-\omega(\tau, s)) \psi(s, x(s)) d s\right)\right\|_{\rho} \\
& \leq \|(\lambda(\tau, x(\tau))-\lambda(\tau, 0)+\lambda(\tau, 0)) \\
& \times\left(\int_{0}^{t}(\omega(t, s)-\omega(\tau, s)) \psi(s, x(s)) d s\right) \|_{\rho} \\
& \leq(a k+h)|\omega(t, 0)-\omega(\tau, 0)|_{L_{\infty}} \int_{0}^{t} \beta(s) \gamma(k) d s \\
& \leq \frac{k}{r}|\omega(t, 0)-\omega(\tau, 0)|_{L_{\infty}}, \\
& \left\|(\lambda(t, x(t))-\lambda(\tau, x(\tau))) \int_{0}^{t} \omega(\tau, s) \psi(s, x(s)) d s\right\|_{\rho} \\
& \leq\left\|(\lambda(t, x(t))-\lambda(\tau, x(\tau))) r \int_{0}^{t} \beta(s) \gamma(k) d s\right\|_{\rho} \\
& \leq \frac{k}{a k+h}\left(\|\lambda(t, x(t))-\lambda(t, x(\tau))\|_{\rho}\right. \\
& \left.+\|\lambda(\tau, x(\tau))-\lambda(t, x(\tau))\|_{\rho}\right) \\
& \leq \frac{k}{a k+h}\left(a\|x(t)-x(\tau)\|_{\rho}+h\right), \\
& \left\|\lambda(\tau, x(\tau)) \int_{\tau}^{t} \omega(\tau, s) \psi(s, x(s)) d s\right\|_{\rho} \\
& =\|(\lambda(\tau, x(\tau))-\lambda(\tau, 0)+\lambda(\tau, 0)) \\
& \times \int_{\tau}^{t} \omega(\tau, s) \psi(s, x(s)) d s \|_{\rho} \\
& \leq(a k+h) r \int_{\tau}^{t} \beta(s) \gamma(k) d s \\
& \leq \frac{k}{b}|t-\tau|,
\end{aligned}
$$

then $S(B)$ is $\|\cdot\|_{\rho}$-equicontinuous. By using the Arzela-Ascoli Theorem, we obtain that $S$ is a $\|\cdot\|_{\rho}$-compact mapping.

We show that $S$ is $\|\cdot\|_{\rho}$-continuous. Suppose that $\varepsilon>0$ is given. We find a $\delta>0$ such that $\|x-y\|_{\sigma}<\delta$. We have

$$
\begin{aligned}
& \|S x(t)-S y(t)\|_{\rho} \\
& =\| \lambda(t, x(t)) \int_{0}^{t} \omega(t, s) \psi(s, x(s)) d s \\
& \quad-\lambda(t, y(t)) \int_{0}^{t} \omega(t, s) \psi(s, y(s)) d s \|_{\rho}
\end{aligned}
$$

$$
\begin{aligned}
\leq & \left\|(\lambda(t, x(t))-\lambda(t, y(t))) \int_{0}^{t} \omega(t, s) \psi(s, x(s)) d s\right\|_{\rho} \\
& +\left\|\lambda(t, y(t)) \int_{0}^{t}(\psi(s, x(s))-\psi(s, y(s))) d s\right\|_{\rho} \\
\leq & \frac{k a}{a k+h}\|x(t)-y(t)\|_{\rho}+(a k+h) r \int_{0}^{t}\|x(s)-y(s)\|_{\rho} d s \\
\leq & \frac{k a}{a k+h}\|x-y\|_{\sigma}+(a k+h) r b\|x-y\|_{\sigma} \\
\leq & \varepsilon .
\end{aligned}
$$

Since $\phi$ is $\|\cdot\|_{\rho}$-continuous, it shows that $T$ transforms $C\left(I, L^{\varphi}\right)$ into itself. In view of supremum $\|\cdot\|_{\rho}$ and condition (1), it is easy to see that $T$ is $\|\cdot\|_{\sigma}$-expansive with constant $l \geq 2$.

$$
\text { For } x, y \in B \text {, }
$$

$$
\begin{aligned}
& \|T x(t)-T y(t)\|_{\rho} \\
& \quad \leq\|x(t)-y(t)\|_{\rho}+\|(I-T) x(t)-(I-T) y(t)\|_{\rho} ;
\end{aligned}
$$

then

$$
\|(I-T) x(t)-(I-T) y(t)\|_{\rho} \geq(l-1)\|x(t)-y(t)\|_{\rho} ;
$$

since $l \geq 2$, then

$$
\|(I-T) x(t)\|_{\rho} \geq(l-1)\|x(t)\|_{\rho} \geq\|x(t)\|_{\rho} .
$$

Now, assume that $x=T x+S y$ for some $y \in B$. Then

$$
\|x(t)\|_{\rho} \leq\|(I-T) x(t)\|_{\rho}=\|S y(t)\|_{\rho} \leq k,
$$

which shows that $x \in B$. Now for each $z \in C\left(I, L^{\varphi}\right)$ we define a map $T_{z}$ as follows:

$$
T_{z}: C\left(I, L^{\varphi}\right) \longrightarrow C\left(I, L^{\varphi}\right)
$$

by

$$
T_{z} x(t)=T x(t)+z(t) ;
$$

for all $x, y \in C\left(I, L^{\varphi}\right)$,

$$
\left\|T_{z} x(t)-T_{z} y(t)\right\|_{\rho}=\|T x(t)-T y(t)\|_{\rho} \geq l\|x(t)-y(t)\|_{\rho} ;
$$

therefore

$$
\left\|T_{z} x-T_{z} y\right\|_{\sigma} \geq l\|x-y\|_{\sigma} ;
$$

then $T_{z}$ is $\|\cdot\|_{\sigma}$-expansive with constant $l \geq 2$ and $T_{z}$ is onto. By Theorem 9, there exists $w \in C\left(I, L^{\varphi}\right)$ such that $T_{z} w=w$; that is, $(I-T) w=z$. Hence $S(B) \subset(I-T)\left(L^{\varphi}\right)$. Therefore by Theorem $12, S+T$ has a fixed point $z \in B$ with $T z+S z=z$; that is, $z$ is a solution of (43).

Finally, some examples are presented to guarantee Theorems 18 and 19. 
Example 20. Consider the following integral equation:

$$
x(t)=\frac{9 x(t)}{1+t^{2}}+\int_{0}^{t} \arctan \left(\frac{5 t(1+s) \sqrt{x(s)}}{(1+t)^{3}(1+\sqrt{x(s)})}\right) d s
$$

where $L^{\varphi}=\mathbb{R}^{+}, I=[0,1]$.

For $x, y \in \mathbb{R}^{+}$and $t \in I$, we have

$$
|\phi(t, x)-\phi(t, y)|=\left|\frac{9 x}{1+t^{2}}-\frac{9 y}{1+t^{2}}\right| \geq \frac{9}{2}|x-y| .
$$

Therefore by Theorem 18, the integral equation (62) has at least one solution.

Example 21. Consider the following integral equation:

$$
x(t)=\frac{9 x(t)}{1+t^{2}}+\frac{1}{8} \arcsin x(t) \int_{0}^{t} \frac{t}{t+s} x(s) d s,
$$

where $\phi(t, x)=\left(9 x /\left(1+t^{2}\right)\right), \lambda(t, x)=(1 / 8) \arcsin x, \omega(t, s)=$ $t /(t+s)$, and $\psi(t, x)=x$. Also $L^{\varphi}=\mathbb{R}^{+}, I=[0,1]$. Therefore by Theorem 19, the integral equation (64) has at least one solution.

\section{Conflict of Interests}

The authors declare that there is no conflict of interests regarding the publication of this paper.

\section{References}

[1] T. Xiang and R. Yuan, "A class of expansive-type Krasnosel'skii fixed point theorems," Nonlinear Analysis: Theory, Methods \& Applications, vol. 71, no. 7-8, pp. 3229-3239, 2009.

[2] T. Xiang and R. Yuan, "Krasnoselskii-type fixed point theorems under weak topology settings and applications," Electronic Journal of Differential Equations, vol. 2010, no. 35, pp. 1-15, 2010.

[3] T. Xiang, "Notes on expansive mappings and a partial answer to Nirenberg's problem," Electronic Journal of Differential Equations, vol. 2013, no. 2, pp. 1-16, 2013.

[4] M. A. Khamsi, "Nonlinear semigroups in modular function spaces," Mathematica Japonica, vol. 37, no. 2, pp. 291-299, 1992.

[5] M. A. Khamsi, W. M. Kozlowski, and S. Reich, "Fixed point theory in modular function spaces," Nonlinear Analysis, vol. 14, pp. 935-953, 1999.

[6] A. Razani and R. Moradi, "Common fixed point theorems of integral type in modular spaces," Bulletin of the Iranian Mathematical Society, vol. 35, no. 2, pp. 11-24, 2009.

[7] A. Hajji and E. Hanebaly, "Fixed point theorem and its application to perturbed integral equations in modular function spaces," Electronic Journal of Differential Equations, vol. 2005, no. 105, pp. 1-11, 2005.

[8] L. Gasiński and N. S. Papageorgiou, Nonlinear Analysis, vol. 9 of Mathematical Analysis and Applications, Chapman \& Hall/ CRC, 2006.

[9] A. Ait Taleb and E. Hanebaly, "A fixed point theorem and its application to integral equations in modular function spaces," Proceedings of the American Mathematical Society, vol. 128, no. 2, pp. 419-426, 2000. 


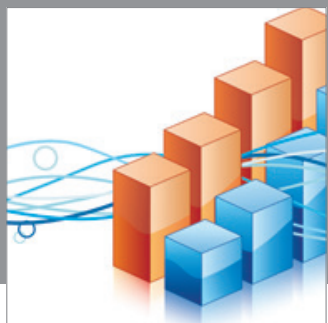

Advances in

Operations Research

mansans

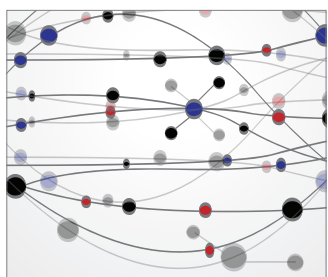

The Scientific World Journal
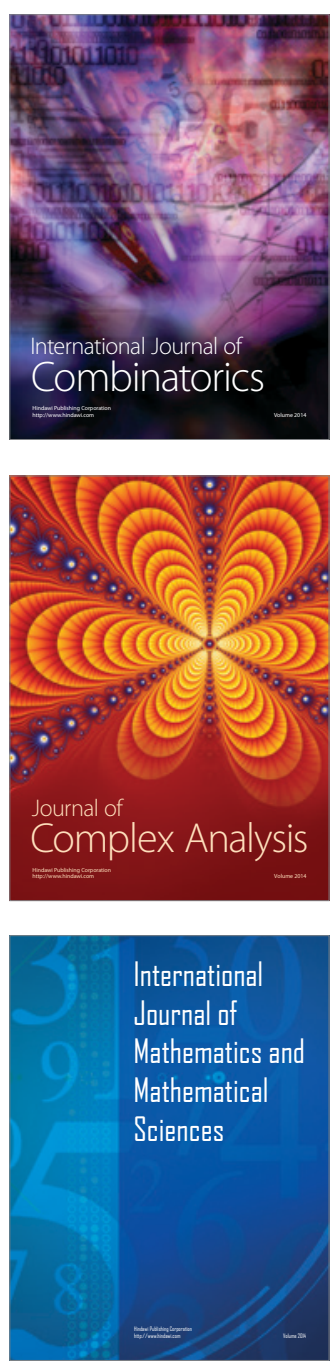
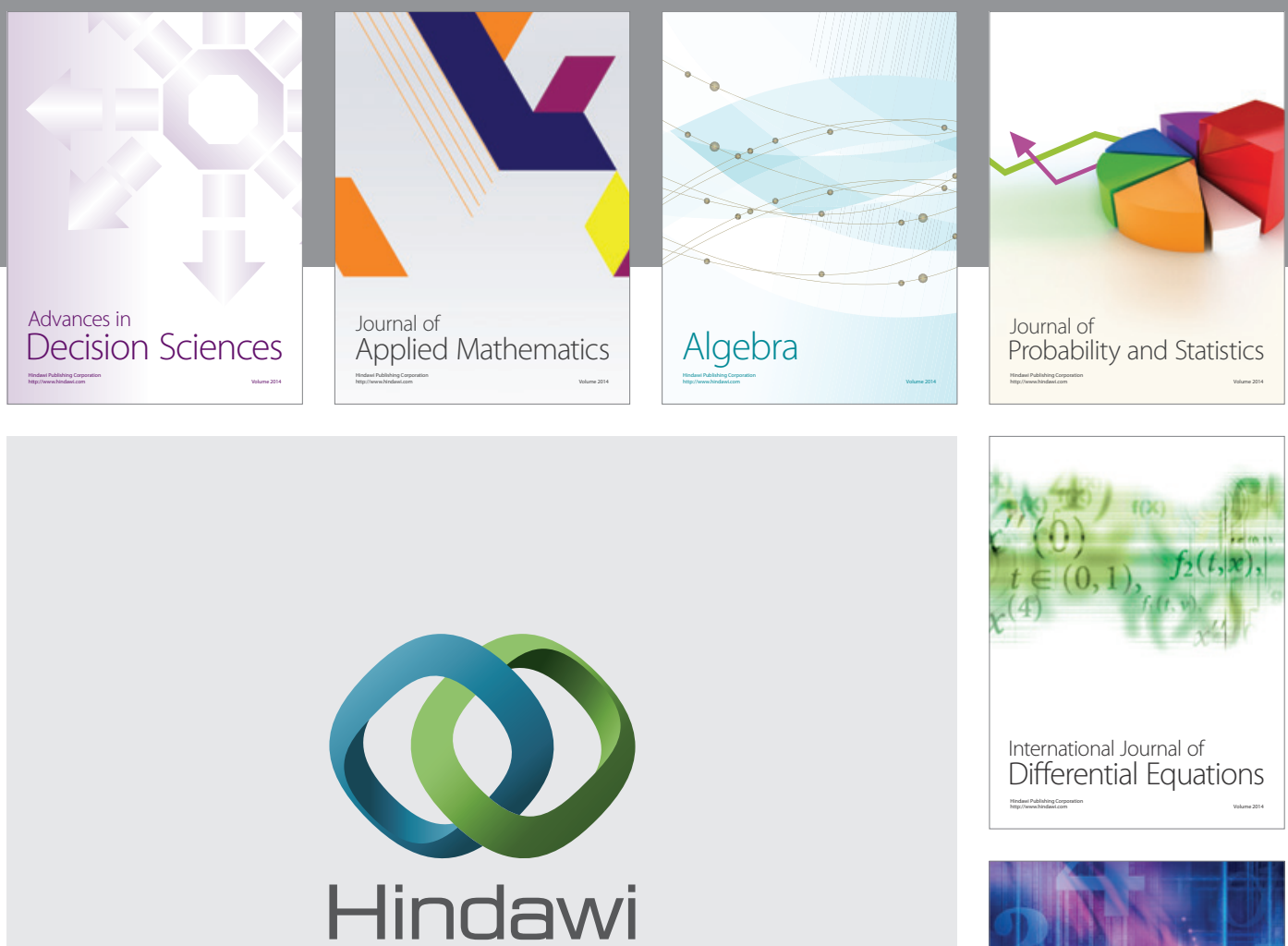

Submit your manuscripts at http://www.hindawi.com
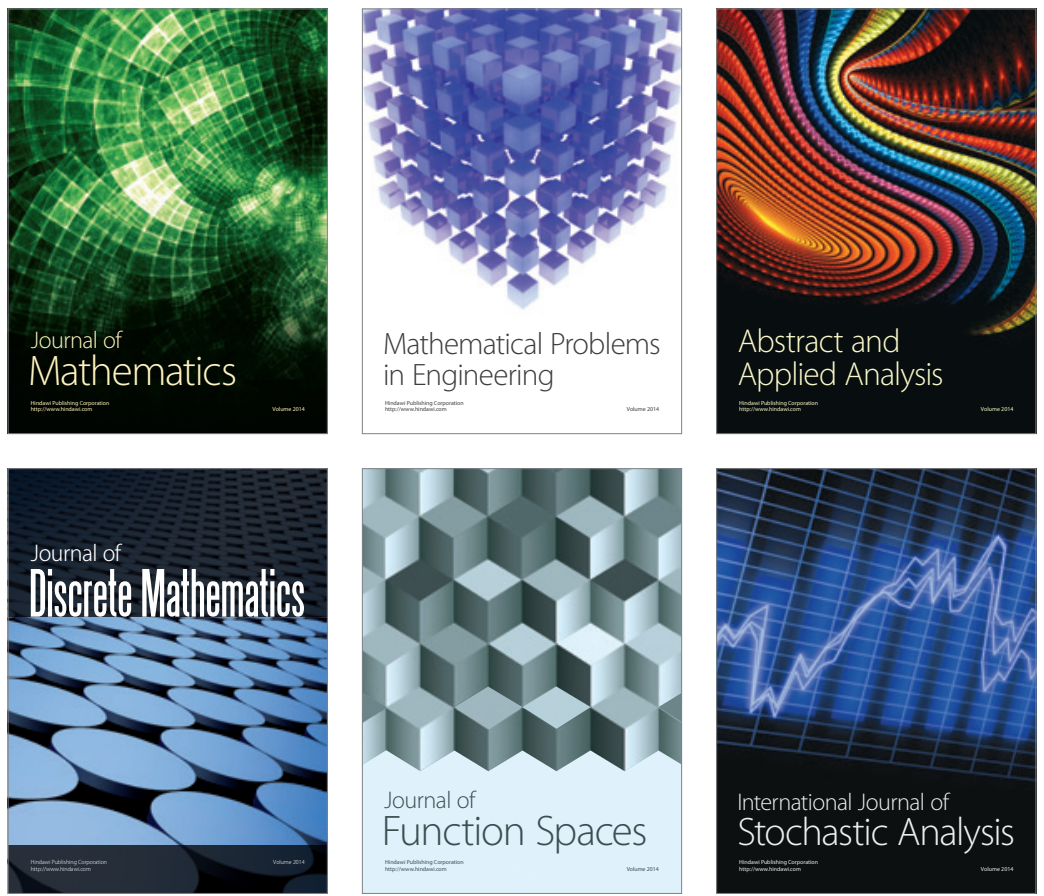

Journal of

Function Spaces

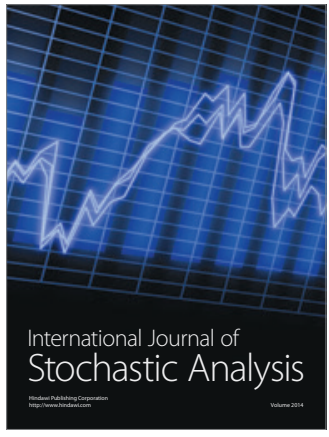

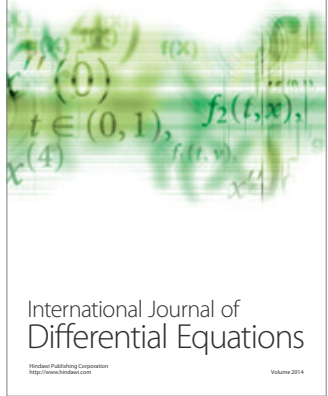
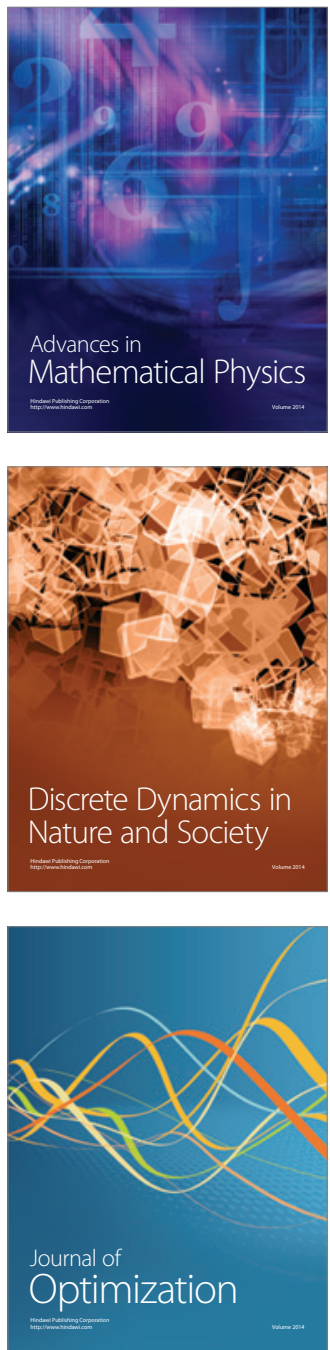